\title{
Fluid Flow and Heat Transfer during the Electron Beam Cold Hearth Re- melting Process
}

\author{
Yaoping Xu, Houfa Shen \\ Key Laboratory for Advanced Materials Processing Technology, Ministry of Education \\ School of Materials Science and Engineering, Tsinghua University \\ Beijing, 100084, China \\ xuyp13@mails.tsinghua.edu.cn; shen@tsinghua.edu.cn
}

\begin{abstract}
Fluid flow and heat transfer plays an important role in Electron Beam Cold Hearth Re-melting (EBCHR or EB Re-melting) process. Because of the asymmetry in geometry of the processing equipment, fluid flow is asymmetrical in the mold which harms the pool sump, the solidification shell and the quality of ingot. Three-dimensional unsteady state model has been built to describe fluid flow and solidification during EBCHR of titanium slab. Mixed Lagrange and Euler (MiLE) method was applied to investigate the unsteady state solidification process. Inhomogeneous surficial power inputs were employed to adjust the fluid flow, temperature distribution and sump shape. The results showed that the surficial mean velocity of fluid descends along the mold centerline and equals to zero when the forced flow encounters the backward flow in the zone away from the inlet. Heat concentration forms in the zone of low surficial fluid velocity. High density power input in the zone near the inlet is efficient to adjust the symmetry of pool sump during EBCHR process.
\end{abstract}

Keywords: Electron Beam Cold Hearth Re-Melting, Fluid Flow, Heat Transfer, Pool Sump.

\section{Introduction}

Titanium is widely used in the aerospace industry. Electron Beam Cold Hearth Re-melting (EBCHR) process has been developed to produce high quality titanium ingots [1]. Fig. 1 shows the schematic of an EBCHR process. The titanium sponge is put into the melting hearth and the high power density electron beam is used to melt raw materials. Then the molten titanium flows into refining hearth, where the purifying process happens. The purified melt is solidified in the copper mold.

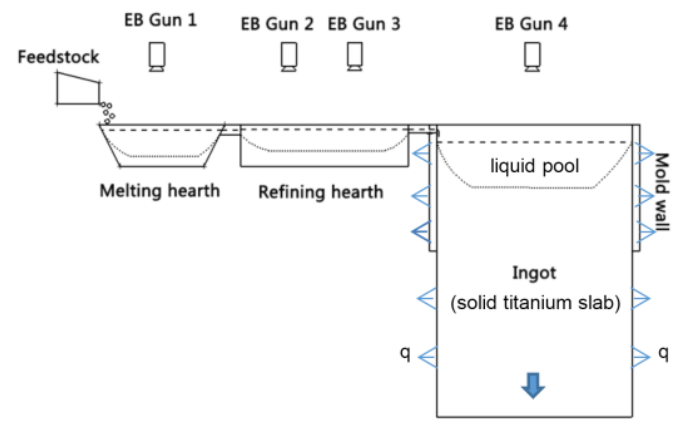

Fig. 1: Schematic of electron beam cold hearth re-melting process.

Top surficial power input in the mold is the most important factor in the EBCHR process. It substantially affects fluid flow and heat transfer which determines the shape of pool sump during the solidification of titanium. Asymmetrical pool sump leads to ingot distortion, while liquid/solid condition of the surface contacting to mold influences the surface quality of ingot. To address these problems, various works including numerical simulation have been done by many researchers. Zhuk et. al. [2] proposed a method to investigate the relationship between electron beam (EB) power and ingot surface quality, consequently, found that a shallow pool depth and solid/liquid between the ingot and the mold determined the surface quality of titanium ingot. Shuster et. al. analyzed the surficial fluid flow and the influence of the thermal-driven 
buoyancy [3]. Zhao et. al. simulated the three-dimensional steady state thermal fluid flow in ingot [4]. However, few works have been done on modeling fluid flow of slab ingots during the EBCHR process [5 7]. The pool depth is more shallow in EBCHR process. Flow field, temperature field and pool sump are very important in slab via EBCHR process. As the demand for high quality titanium ingot grows, emphasis on reducing surface defeat becomes more and more urgent. Concrete discrimination of the pool sump and temperature distribution for the EBCHR slab is needed.

In this work, Mixed Lagrange and Euler (MiLE) method is employed to simulate the unsteady solidification process which can exactly describe the sump shape of EBCHR ingot. A set of power input strategies is employed to analyze the flow attenuation, heat concentration and sump shape within the mold.

\section{Mathematical Model}

\subsection{Model and Materials Properties}

The unsteady state model, named MiLE method is proposed to solve the heat and momentum transportation duirng the EBCHR using ProCAST software. Hexahedral mesh is employed for the modeling of finite element method. The calculation domain for 3-D heat transfer and fluid flow analysis in titanium slab is shown in Fig. 2. A 10 mm height Euler mesh is set as the up domain while a $20 \mathrm{~mm}$ height Lagrange mesh is set as the down domain for unsteady state analysis. 200 layers with each layer height of $10 \mathrm{~mm}$ are set between the up domain and the down domain. The cross-section of the slab ingot is $1050 \times 210 \mathrm{~mm}$. The copper mold is open in the top and the bottom with the internal cavity identical to the external profile of the slab. The height of mold is $600 \mathrm{~mm}$ with a rectangular inlet closed to narrow face.
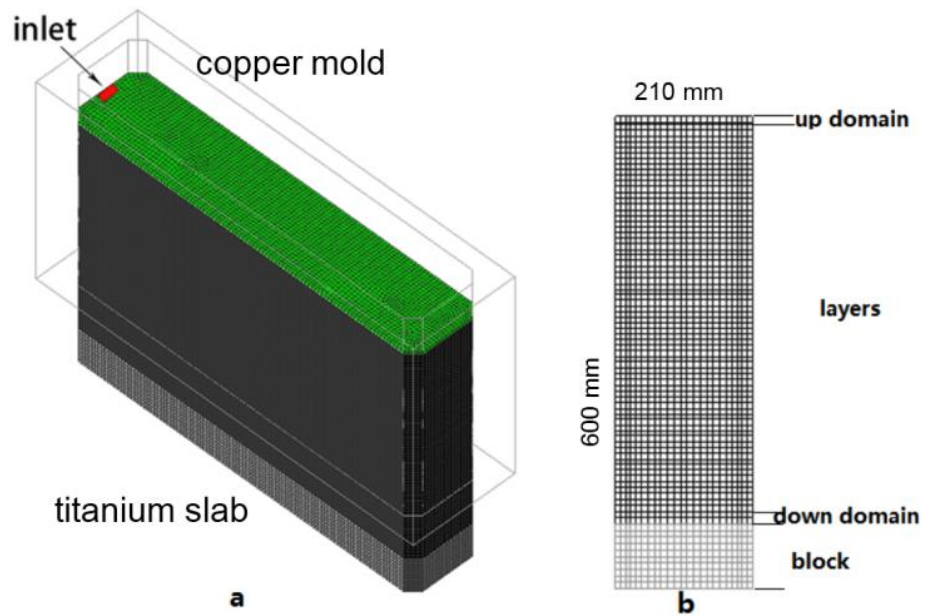

Fig. 2: (a) Schematic of calculation domain with MiLE method, (b) including "up domain", "layers" and "down domain".

Material's properties for simulation were calculated from ProCAST database using Scheil model.

\subsection{Power Input on Mold Top Surface}

Heat flux is used to represent the EB gun power on the liquid surface of titanium slab. For applying a distinct power input, the top surface is sectioned to 6 zones as shown in Fig. 3.

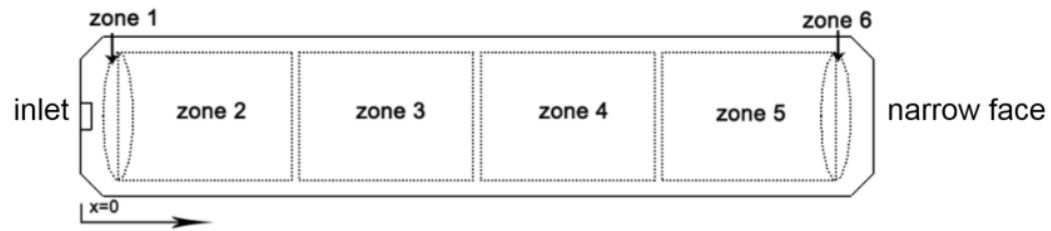

Fig. 3: Four rectangular zones and two elliptical zones for electron beam action in on the melt top surface. 
The size of zone 2, 3, 4 and 5 is same of $230 \times 190 \mathrm{~mm}$, with $10 \mathrm{~mm}$ away from the mold wall. In order to avoid quick solidification of melt at four corners located at the mold narrow face, elliptical zone 1 and zone 6 are designed near the corners. Electron beams are programed to scan the prescribed pattern in six zones in sequence, and power densities are controlled by distributing a series of scanned points on the surface of melt. Considering the losses of electron beam power by scattering, absorption factor is set to 0.7 .

Table 1: Strategy of EB gun power distribution percentage and total input on mold top surface.

\begin{tabular}{|c|c|c|c|c|c|c|c|}
\hline case & $\begin{array}{c}\text { zone 1 } \\
(\%)\end{array}$ & zone 2 (\%) & zone 3 (\%) & zone 4 (\%) & zone 5 (\%) & zone 6 (\%) & total power (kW) \\
\hline 1 & 14 & 18 & 18 & 18 & 18 & 14 & 267 \\
\hline 2 & 14 & 22 & 22 & 14 & 14 & 14 & 267 \\
\hline
\end{tabular}

\subsection{Boundary and Initial Conditions}

A rectangular inlet is incorporated on the top surface near the mold narrow face as shown in Fig. 3 . The size of the inlet is $15 \times 20 \mathrm{~mm}$, and the inlet temperature of melt is assumed to be $1900{ }^{\circ} \mathrm{C}$. The vertical inlet velocity is assumed to be $145 \mathrm{~mm} / \mathrm{s}$, according to the casting speed of $2 \times 10^{-4} \mathrm{~m} / \mathrm{s}$. Heat transfer coefficient between the mold and the slab is determined linearly varying from $500 \mathrm{~W} /\left(\mathrm{m}^{2} \cdot \mathrm{K}\right)$ at melt top to $30 \mathrm{~W} /\left(\mathrm{m}^{2} \cdot \mathrm{K}\right)$ at mold bottom. This coefficient is determined according to the practice and references [3,4,6,7]. Low heat transfer coefficient is determined because the internal face of the mold is rough and covered with titanium from evaporation. Air gap between the slab and mold forms rapidly due to the shrinkage of solidified slab in EBCHR. In industrial production, a $5 \mathrm{~mm}$ to $10 \mathrm{~mm}$ width gap is usually found at the bottom of the mold, indicating by the solidified overflow in the air gap. After gap formation, the heat transfer coefficient within the mold is calculated using the following expression:

$$
\mathrm{h}=\frac{1}{\frac{1}{h_{0}}+R_{g a p}}
$$

where $h\left(\mathrm{~W} /\left(\mathrm{m}^{2} \cdot \mathrm{K}\right)\right)$ is the adjusted heat transfer coefficient, $h_{0}\left(\mathrm{~W} /\left(\mathrm{m}^{2} \cdot \mathrm{K}\right)\right)$ is the initial value of the heat transfer coefficient (equal to $\left.500 \mathrm{~W} /\left(\mathrm{m}^{2} \cdot \mathrm{K}\right)\right), R_{\text {gap }}(\mathrm{W} /(\mathrm{m} \cdot \mathrm{K}))$ is the heat resistance between the gap, which is calculated using the following expression:

$$
R_{g a p}=\frac{1}{\frac{k}{g a p}+h_{r a d}}
$$

where $k(\mathrm{~W} /(\mathrm{m} \cdot \mathrm{K}))$ is the conductivity of air, gap is the air gap width, $h_{\mathrm{rad}}\left(\mathrm{W} /\left(\mathrm{m}^{2} \cdot \mathrm{K}\right)\right)$ is the equivalent radiative heat transfer coefficient.

The heat transfer coefficient in the region below the mold is equal to constant of $10 \mathrm{~W} /\left(\mathrm{m}^{2} \cdot \mathrm{K}\right)$. The external face of the copper mold is set as water cooling condition. Because of unformly contact, the heat transfer coefficient between slab bottom and block is set to be $200 \mathrm{~W} /\left(\mathrm{m}^{2} \cdot \mathrm{K}\right)$. Mold domain is assumed to be at a room temperature of $25{ }^{\circ} \mathrm{C}$.

\section{Results and Discussion}

\subsection{Fluid Flow}

Fig. 4 (b) and (c) show the flow velocity of melt in the vertical and horizontal sections of slab with identical EB power input in four surface zones (zone 2 5) as indicated in Fig. 4 (a). Fig. 4 (b) illustrates that flow direction on top surface and on the bottom of the sump is away from inlet. It shows that horizontal flow forms a belt-liked confluence on the top of melt, and then it is interrupted at the distance to the inlet $x=750 \mathrm{~mm}$. This is mainly because that the fluid flow with opposite direction encounters in this zone. The flow velocity on the top surface decreases with the increasing distance from inlet. 


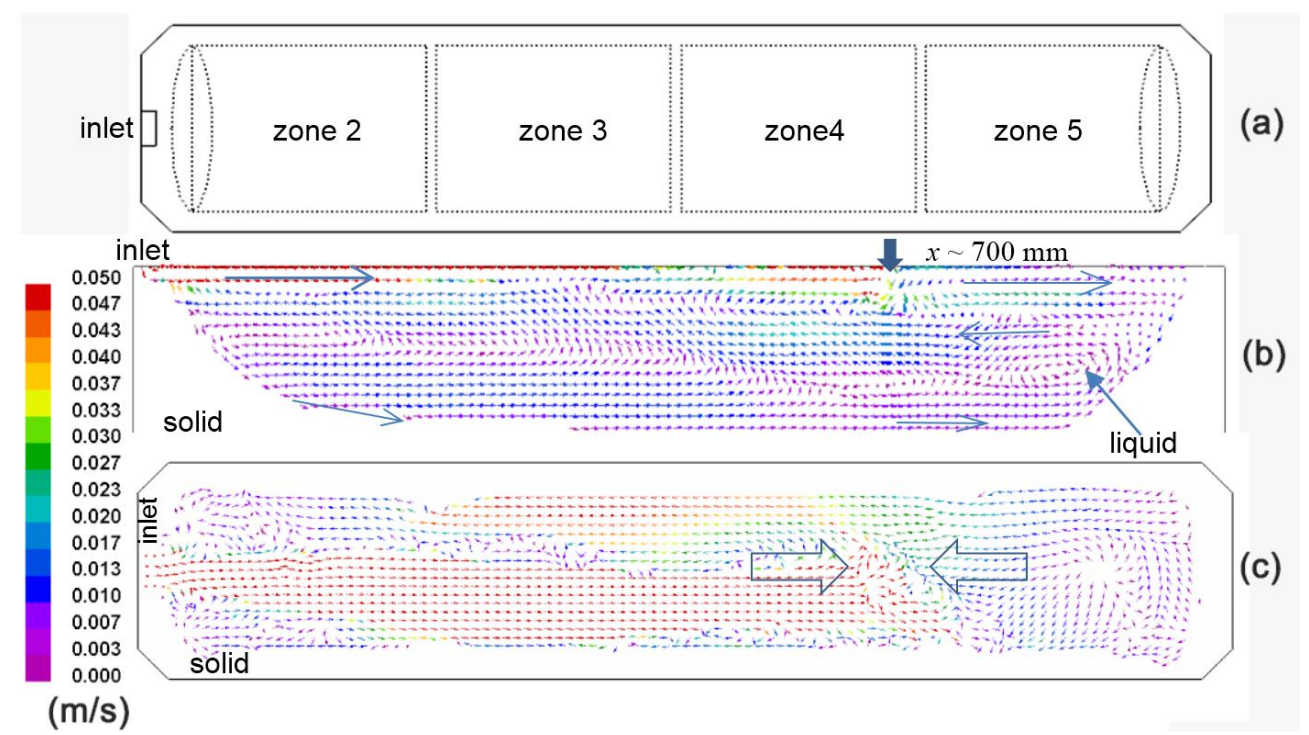

Fig. 4: (a) Distribution of power input in mold horizontal surface; (b) flow velocity in vertical section; (c) flow velocity in horizontal section of slab.

\subsection{Heat Transfer and Solidification}

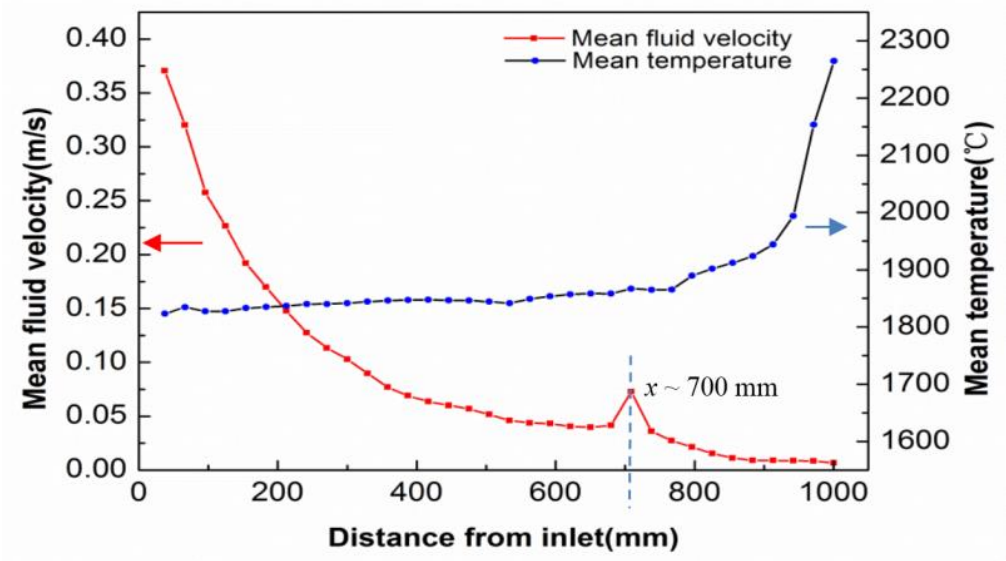

Fig. 5: Mean fluid velocity and mean temperature distribution along the centerline of the top surface with identical EB gun power input in zone 2 to zone 5 .

Fig. 5 shows the mean temperature and the mean fluid velocity on the top surface of slab. With the increasing distance to the inlet, the surficial mean temperature increases while the mean fluid velocity generally decreases. The mean temperature increases slowly to $1900{ }^{\circ} \mathrm{C}$ then accelerates in zone $4(x \sim 700 \mathrm{~mm})$. It is in the zone 6 that the mean temperature on the top surface raises to a supreme temperature of $2333{ }^{\circ} \mathrm{C}$. It is easy to understand the temperature variation according to the effects of fluid flow as shown in Fig. 4. Strong flow with direction away from the inlet can transport high temperature melt to the zone opposite to the inlet. The descending of mean fluid velocity leads heat concentration on the top surface because of attenuated flow's poor capacity of heat transfer. The spark on the mean fluid velocity curve is related to the conflict of fluid flow with different directions.

Because of the asymmetry of mold with the inlet in one side, the coupling of fluid flow and heat transfer leads the asymmetry of liquid sump in titanium slab. Accordingly, this asymmetry in sump shape can be adjusted by optimizing the power distribution on the mold surface. Fig. 6 presents the pool sump before and after the optimization of the power. Case 1 in Fig. 6 presents pool sump with symmetrical power input (Table 1) while case 2 presents pool sump 
with high density power input in zone near the inlet. In order to keep symmetrical pool sump during the EBCHR process, high density surficial power input is needed in the zone near the inlet because the heat around the inlet can be transported by the surficial fluid flow. The calculated surface temperature of melt and the predicted shape of pool sump are in agreement with the practice.

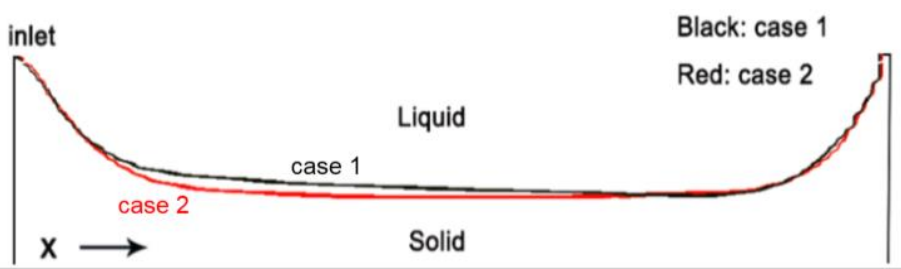

Fig. 6: Comparison of pool sump along centerline under symmetrical power input (case 1) and asymmetrical power input (case 2).

\section{Conclusions}

Three-dimensional unsteady state model has been proposed to describe fluid flow and heat transfer during the Electron Beam Cold Hearth Re-melting (EBCHR) process of titanium. A set of power input density is used to evaluate the effect of coupling of fluid flow and heat transfer on the sump profile of EBCHR slab. The following conclusions are drawn:

1) The direction of fluid velocity on top surface and at the bottom of sump remains pointing to the mold narrow face opposite to the inlet. The forced flow from inlet and the backward flow from opposite encounters beneath the top surface of melt at the position of $750 \mathrm{~mm}$ away from the inlet.

2) The surficial mean temperature of melt increases slowly near the inlet and rises rapidly away from the inlet. Heat concentration forms in the position near the mold narrow face opposite to the inlet due to the low surficial flow velocity and heat transfer. Both the calculated temperature and the shape of pool sump are consistent with the measurements.

3) In order to keep the symmetrical pool sump during EBCHR process, high density surficial power input is needed in the region near the inlet.

\section{Acknowledgement}

This work was financially supported by the national key research and development program of China (2016YFB0301202).

\section{References}

[1] A. Mitchell, "Melting, casting and forging problems in titanium alloys," Materials Sci and Eng A. vol. 243, no. 1-2, pp. 257-262, 1998.

[2] H. V. Zhuk, P. A. Kobryn, and S. L. Semiatin, "Influence of heating and solidification conditions on the structure and surface quality of electron-beam melted Ti-6Al-4V ingots," J. Materials Processing Tech., vol. 190, pp. $387-$ 392, 2007.

[3] R. Shuster, C. Reilly, D. M. Maijer, S. L. Cockcroft, "Modeling and optimizing Ti-6Al-4V ingot production," in IOP Conference Series: Materials Science and Engineering, vol. 33, 012005, pp. 1-8, 2012.

[4] X. Zhao, C. Reilly, L. Yao, D. M. Maijer, S. L. Cockcroft and J. Zhu, "A three-dimensional steady state thermal fluid model of jumbo ingot casting during electron beam re-melting of Ti-6Al-4V," Applied Mathematical Modelling. vol. 38, no. 14, pp. 3607-3623, 2014.

[5] K. I. Suzuki and S.Watakabe, "Melting and precision casting of Ti-6Al-4V alloy by use of electron beam furnace," Metals and Materials Inter., vol. 9, no. 4, pp. 359-367, 2003.

[6] P. A. Kobryn, and S. L. Semiatin, "Determination of interface heat-transfer coefficients for permanent-mold casting of Ti-6Al-4V," Metallurgical and Materials Trans. B., vol. 32B, pp. 685-695, 2001.

[7] K. Vutova and V. Donchev, "Non-stationary heat model for electron beam melting and refining - an economic and conservative numerical method," Applied Mathematical Modelling, vol. 40, no. 2, pp. 1565-1575, 2016. 